\title{
Predictive Antiwindup Compensation for Repetitive Control Supporting Nanoscanning
}

\author{
Peng Yan $^{\circledR}$, Senior Member, IEEE, Pengbo Liu ${ }^{\circledR}$, and Hitay Özbay ${ }^{\circledR}$, Fellow, IEEE
}

\begin{abstract}
Ultrahigh precision scanning has emerged as a promising and challenging technology in nanoscale measurement and manipulation. Besides the requirement of dynamical tracking of periodical references, the existence of control saturation, due to the limited stroke of microactuators, poses additional challenges for the control of such systems. To address this problem, we propose a repetitive control structure with predictive antiwindup compensation to support nanoscale repetitive tracking/scanning with a minimized impact of actuator saturation. In particular, the proposed antiwindup compensator is activated by the time-lead control output ahead of saturation due to the time-delay block in the repetitive control structure. According to the input/output (I/O)-based equivalent representation and sector bound criterion, stability conditions for the antiwindup compensator are derived, which are further formulated as an $H_{\infty}$ optimization problem with the robustness against model uncertainties, where the optimal compensator is developed. The effectiveness of the proposed tracking control architecture is further verified in real-time experiments on a piezoelectricactuator-driven nanostage, where significant improvements are demonstrated comparing with the existing results.
\end{abstract}

Index Terms-Antiwindup control, $H_{\infty}$ optimization, nanotracking, repetitive control, time delay.

\section{INTRODUCTION}

$\mathbf{N}$ ANOSCALE scanning has been recognized as one of the key enabling technologies in nanomeasurement and manipulation instruments, such as atomic force microscope (AFM) and scanning tunneling microscope (STM) [1]-[3]. The accuracy and speed of the scanning motions fundamentally determine the quality and efficiency of these instruments. In past decades, most of the research efforts in the area of nanoservo systems have been devoted to nanopositioning (see [4]-[6] and references therein). With

Manuscript received May 11, 2020; accepted July 9, 2020. Date of publication July 30, 2020; date of current version June 10, 2021. Manuscript received in final form July 19, 2020. This work was supported in part by the Natural Science Foundation of Shandong Province under Grant ZR2019ZD08, in part by the National Natural Science Foundation of China under Grant 51775319 and Grant 51905287, in part by the Science and Technology Project of Shenzhen City under Grant JCYJ20180305164242690, and in part by the Key Research and Development Program of Shandong Province under Grant 2018GGX103009435. Recommended by Associate Editor K. Barton. (Corresponding author: Peng Yan.)

Peng Yan is with the Key Laboratory of High-efficiency and Clean Mechanical Manufacture, Ministry of Education, School of Mechanical Engineering, Shandong University, Jinan 250061, China (e-mail: pengyan2007@gmail.com).

Pengbo Liu is with the School of Mechanical and Automotive Engineering, Shandong Academy of Sciences, Qilu University of Technology, Jinan 250353, China (e-mail: pengbo@qlu.edu.cn).

Hitay Özbay is with the Department of Electrical and Electronics Engineering, Bilkent University, 06800 Ankara, Turkey (e-mail: hitay@ bilkent.edu.tr).

Color versions of one or more of the figures in this article are available online at https://ieeexplore.ieee.org.

Digital Object Identifier 10.1109/TCST.2020.3010952 the emerging demands on scanning applications, the design and control problems associated with nanotracking attract significant research in recent years [7]-[9].

Note that during the operation of nanoscanning, the motion system is required to precisely follow specific repetitive signals, such as the triangular wave. To address the challenge of dynamical tracking of periodic references, the repetitive control methods were developed with successful applications in motion systems [10]-[12]. In such a control structure, high accuracy tracking can be achieved by introducing a time-delay element corresponding to the reference period in the positive feedback loop, which leads to an infinite-dimensional internal model whose frequency response presents resonance peaks in all integer multiples of the fundamental frequency of the periodic signal [10]. In particular, the repetitive control method has been introduced to the control of nanostages in recent literature, such as [13] and [14].

It should be noticed that the direct implementation of repetitive controllers on nanoscanning can easily trigger actuator saturation due to the microscale stroke of piezoelectric actuators in the nanostages, where the existence of multiple integrators involved in the repetitive controller [15] deteriorates the saturation issue, which may have significant adverse effects on system performance and possible mechanical failures [16]. Therefore, nanoscanning control in the presence of actuator saturation is still a challenging and open problem.

Various control approaches have been explored to deal with the saturation nonlinearities, e.g., parametric discrete-time periodic Lyapunov equation-based method [17], nested switching control [18], antiwindup compensation [19], [20], and internal model compensation [21], to name just a few. Note that most of the existing results on saturation control are discussed in a general control framework without considering the specific structure of the repetitive control, which cannot be used to optimize the compensation performance for tracking purposes. It is also worth noting that several representative results investigated the antiwindup problem in a repetitive controller setting [15], [22], [23], where antiwindup mechanisms are taking effect once the actuator saturation occurs. However, recent results [19], [24]-[26] indicate that activating the antiwindup mechanism in anticipation of actuator saturation may lead to significant improvement of tracking performance in comparison with the immediate antiwindup design.

Due to the time-delay block in the repetitive control structure, it is possible to obtain the time lead control output and compensate for early saturation before it is occurring. With this motivation, we propose a predictive antiwindup design taking advantage of the repetitive control structure, which 


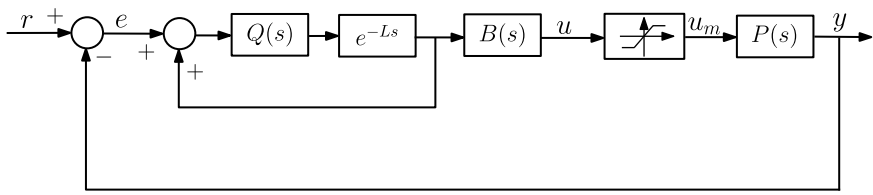

Fig. 1. Repetitive control structure with input saturation.

allows for the tracking of periodical signals in the presence of actuator saturation. Different from the existing anticipatory antiwindup schemes with virtual saturation element with reduced limit [19], [24]-[26], the proposed antiwindup design achieves anticipatory compensation of saturation on top of the predicted controller output. Real-time experiments implemented on a piezo-driven nanostage demonstrate that the proposed control architecture significantly improves the scanning performance and saturation compensation capability compared with representative results in the literature.

The rest of this brief is organized as follows. In Section II, the repetitive control structure is briefly discussed, and the saturation problem is formulated. The predictive antiwindup design on top of the repetitive controller is proposed with a detailed design procedure in Section III. Real-time experiments are conducted on a nanostage in Section IV to verify the performance enhancement of the proposed control structure, followed by some concluding remarks in Section V.

\section{Problem Formulation}

\section{A. Preliminaries on Repetitive Control}

We first recall the basic repetitive control structure [10] shown in Fig. 1, where $P(s)$ is the control plant (assumed to be stable and minimum phase ${ }^{1}$ ). The repetitive control structure includes a time-delay unit in a positive inner feedback loop such that the repetitive control is capable of tracking periodic signals with a fixed period $L$.

In repetitive control applications, $Q(s)$ is selected as a unity low-pass filter to limit the bandwidth of the signal model and guarantee the stability of system, and $B(s)$ is designed to approximate the inverse of plant to obtain a good performance. Theoretically, the design requires both $B(s) P(s)$ and $Q(s)$ to be ideal unity low-pass filters with very sharp roll-off [11]

$$
\begin{aligned}
B(j \omega) P(j \omega) & \approx 1 \quad \forall \omega \leq \omega_{b} \\
B(j \omega) P(j \omega) & \approx 0 \quad \forall \omega>\omega_{b} \\
Q(j \omega) & \approx 1 \quad \forall \omega \leq \omega_{q} \\
Q(j \omega) & \approx 0 \quad \forall \omega>\omega_{q} .
\end{aligned}
$$

The cutoff frequencies $\omega_{q}$ and $\omega_{b}$ are determined from performance and stability robustness requirements. We shall see that to achieve these requirements, we need $\omega_{q} \ll \omega_{b}$.

For the cases without actuator saturation, the repetitive controller can be designed to guarantee the bounded-input bounded-output (BIBO) stability of the closed-loop system [11], with the capability of tracking periodic signals composed of various harmonics of base frequency $1 / L$. Therefore,

\footnotetext{
${ }^{1}$ It is a viable assumption, which can be realized by a stabilizer if the original system does not satisfy this condition [10].
}

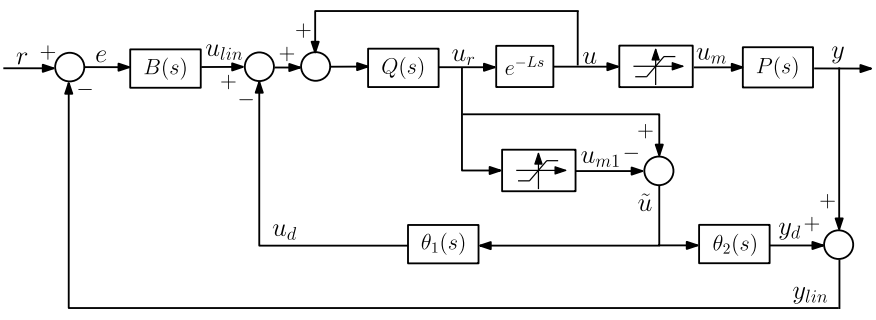

Fig. 2. Repetitive control structure with predictive antiwindup compensator.

the repetitive control method has been successfully applied to the periodical scanning of nanostages [27], [28].

\section{B. Control Saturation Issues}

Due to the microscale stroke of piezoelectric actuators in the nanostages, control saturation is frequently experienced in tracking/scanning applications, where the integral property of the controllers (mainly for the purpose of low-frequency disturbance rejection) deteriorates the saturation issue.

In the presence of actuator saturation, the control signals are constrained by

$$
u_{m}=\operatorname{sat}(u)= \begin{cases}\sigma_{1}, & u<\sigma_{1} \\ u, & \sigma_{1} \leq u \leq \sigma_{2} \\ \sigma_{2}, & u>\sigma_{2}\end{cases}
$$

where $u_{m}$ is the actual control input to the plant, as shown in Fig. $1, u$ is the controller output, and $\sigma_{1}$ and $\sigma_{2}$ are the saturation limits. Note that the piezoelectric actuators in nanostages usually have asymmetric voltage limits moving forward or backwards. Therefore, $\left|\sigma_{1}\right|$ and $\left|\sigma_{2}\right|$ are usually different for such systems.

The difference between the controller outputs and the actual plant inputs may result in significant performance degradations, such as slow and oscillatory transients, as well as stability issues [23]. In the sequel, a novel antiwindup compensation scheme for repetitive controllers is developed to handle the saturation nonlinearity. Due to the time-delay element in the repetitive control structure, the proposed antiwindup mechanism can be activated in anticipation of actuator saturation to improve the saturation compensation capability and the tracking performance.

\section{Antiwindup Compensation Design}

We propose a predictive antiwindup compensation structure on top of the repetitive controller, as shown in Fig. 2, where the antiwindup compensators $\theta_{1}(s)$ and $\theta_{2}(s)$ adjust the controller outputs and system outputs, similar to the antiwindup framework proposed in [16]. Compared with existing results, a major innovation of the proposed compensators is the effective use of saturation anticipation by taking advantage of the time-delay unit $e^{-L s}$ in the inner loop of the repetitive controller. Due to the existence of time delay $e^{-L s}$ in the inner loop of the repetitive controller, the signal $u_{r}$ can be regarded as the time-lead control output $u$, which offers the opportunity to predict possible saturation. In order to achieve 


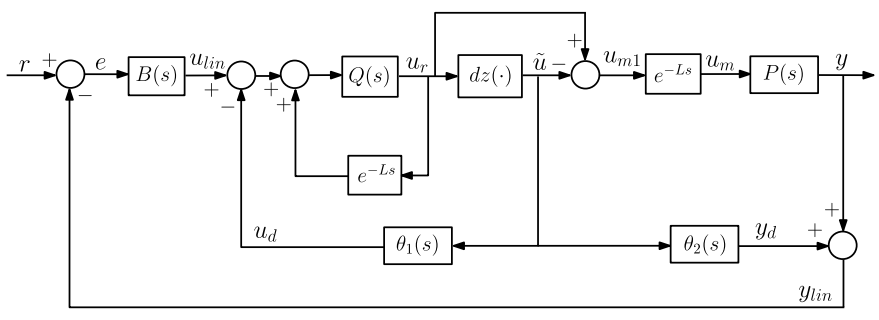

Fig. 3. Equivalent representation of the predictive antiwindup structure with dead-zone operator.

antiwindup compensations in anticipation of actuator saturation, an additional saturation function with the same saturation limit, as shown in (5) (i.e., $u_{m 1}=\operatorname{sat}\left(u_{r}\right)$ ), is introduced in the control loop. The antiwindup compensators $\theta_{1}(s)$ and $\theta_{2}(s)$ are activated by the difference between $u_{r}$ and $u_{m 1}$ to adjust the controller outputs and system outputs ahead of actual saturation occurrence, for better handling of saturation. In what follows, we would like to detail the design procedure of the proposed predictive antiwindup compensators for repetitive control structures.

\section{A. Equivalent Transformation of Control Structure}

Referring to the design method for antiwindup compensators [17], [29], [30], we first formulate the antiwindup compensator design as a control problem with dead-zone nonlinearities. To this end, we have the following condition according to input/output (I/O) relationships.

Proposition 1: The closed-loop control system with two saturation blocks shown in Fig. 2 can be transformed into a control system with one dead-zone block shown in Fig. 3 if the following relationship is satisfied:

$$
\theta_{2}(s)=P(s)\left(1+\tilde{\theta}_{1}(s)\right)
$$

where

$$
\tilde{\theta}_{1}(s)=\frac{Q(s) \theta_{1}(s)}{1-Q(s) e^{-L s}}
$$

Proof: According to the proposed control structure shown in Fig. 2, we have the following relationships under the condition of (6):

$$
\begin{aligned}
y_{\operatorname{lin}} & =\frac{P(s) Q(s)}{1-Q(s) e^{-L s}} u_{\operatorname{lin}}+P(s)\left(u_{m}-u_{m 1}\right) \\
u_{r} & =\frac{Q(s)}{1-Q(s) e^{-L s}}\left(u_{\mathrm{lin}}-\theta_{1}(s) \tilde{u}\right) \\
\tilde{u} & =d z\left(u_{r}\right)= \begin{cases}u_{r}-\sigma_{1}, & u_{r}<\sigma_{1} \\
0, & \sigma_{1} \leq u_{r} \leq \sigma_{2} \\
u_{r}-\sigma_{2}, & u_{r}>\sigma_{2}\end{cases} \\
y_{d} & =\theta_{2}(s) \tilde{u}
\end{aligned}
$$

where $d z(\cdot)$ is the dead-zone operator.

It is worth pointing out that the delay can commute with the saturation operator, that is

$$
u_{m}=\operatorname{sat}(u)=\operatorname{sat}\left(e^{-L s} u_{r}\right)=e^{-L s} \operatorname{sat}\left(u_{r}\right)=e^{-L s} u_{m 1} .
$$

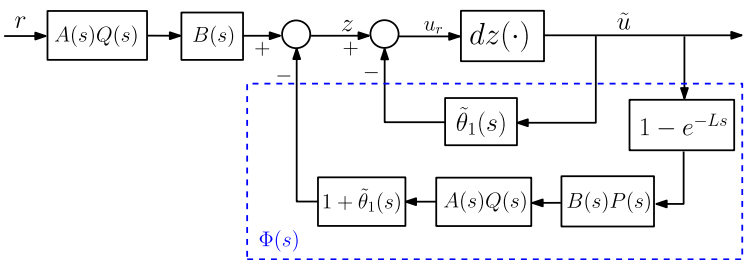

Fig. 4. Simplification of the block diagram in Fig. 3.

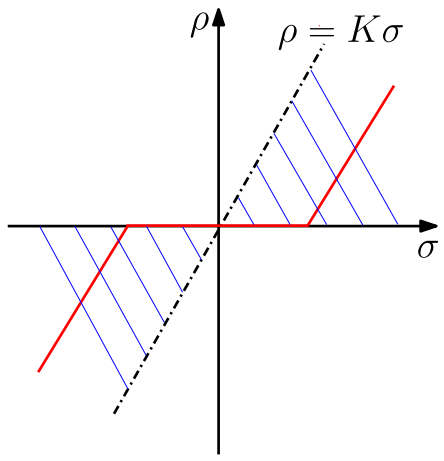

Fig. 5. Dead-zone operator.

Substituting (12) into (8), we derive

$$
y_{\text {lin }}=\frac{P(s) Q(s)}{1-Q(s) e^{-L s}} u_{\text {lin }}+P(s)\left(1-e^{-L s}\right)\left(\tilde{u}-u_{r}\right)
$$

which is identical to the I/O relationship in Fig. 3 and completes the proof.

\section{B. Stability Condition}

According to the I/O relationship, the control structure depicted in Fig. 3 can be further simplified to Fig. 4, where

$$
A(s)=\frac{1}{1-Q(s) e^{-L s}(1-B(s) P(s))} .
$$

Note the fact that $A(s)$ is stable if $B(s)$ and $Q(s)$ satisfy conditions in (1)-(4) as long as $\omega_{q} \ll \omega_{b}$. Accordingly, the stability of the original control structure can be determined by the feedback structure composed of the dead-zone nonlinearity, which can be guaranteed by the following theorem.

Theorem 1: The feedback structure (as shown in Fig. 4) with the dead-zone nonlinearity is stable if there exists an $\varepsilon>0$ such that

$$
\operatorname{Re}\left\{\begin{array}{c}
(1+\varepsilon s)\left[\tilde{\theta}_{1}(s)+A(s) Q(s) B(s) P(s)\right. \\
\left.\left(1-e^{-L s}\right)\left(1+\tilde{\theta}_{1}(s)\right)\right]
\end{array}\right\}+\frac{1}{K}>0
$$

where $s=j \omega, \omega \in(0,+\infty)$.

Proof: Recall the definition of the dead-zone operator in (10). The dead-zone nonlinearity fulfills a sector condition

$$
\forall \sigma \in \mathbb{R}, \quad d z(\sigma)(K \sigma-d z(\sigma)) \geq 0
$$

where $K=1$ and $\rho=K \sigma$ is the boundary of the sector domain, as shown in Fig. 5.

Referring to the Popov criterion [31], [32], the feedback structure composed of $\Phi(s)$ and $d z(\cdot)$ is stable if there exists an $\varepsilon>0$ such that $(1+\varepsilon s) \Phi(s)+1 / K$ is positive real, where

$$
\Phi(s)=\tilde{\theta}_{1}(s)+A(s) Q(s) B(s) P(s)\left(1-e^{-L s}\right)\left(1+\tilde{\theta}_{1}(s)\right) .
$$

This completes the proof. 
According to the theoretical design of $Q(s)$ and $B(s)$, we would like to examine Theorem 1 in the following three cases.

1) For $\omega>\omega_{b}$, we have $B(j \omega) P(j \omega)=0, Q(j \omega)=0$, and $A(j \omega)=1$. In this case, (15) holds if

$$
\left|(1+j \varepsilon \omega) \tilde{\theta}_{1}(j \omega)\right|<1, \quad \forall \omega>\omega_{b} .
$$

2) For $\omega_{q}<\omega \leq \omega_{b}$, we have $B(j \omega) P(j \omega)=1, Q(j \omega)=$ 0 and $A(j \omega)=1$. In this case, (15) holds if

$$
\left|(1+j \varepsilon \omega) \tilde{\theta}_{1}(j \omega)\right|<1, \quad \forall \omega_{q}<\omega \leq \omega_{b} .
$$

3) For $\omega \leq \omega_{q}$, we have $B(j \omega) P(j \omega)=1, Q(j \omega)=1$, and $A(j \omega)=1$. In this case, (15) holds if

$$
\left|M(\omega)(1+j \varepsilon \omega) \tilde{\theta}_{1}(j \omega)\right|<1, \quad \forall \omega \leq \omega_{q}
$$

where

$$
M(\omega)=\left|\frac{2-\cos (L \omega)+j \sin (L \omega)}{2-\cos (L \omega)-\varepsilon \omega \sin (L \omega)}\right| .
$$

It is a simple exercise to show that if $\varepsilon \leq 1 /\left(3 \omega_{q}\right)$, we have $M(\omega) \leq 3 / 2$ for all $\omega \leq \omega_{q}$ (in fact, for a fixed $\omega_{q}$, as $\varepsilon \rightarrow 0$, we have $\left.\max _{0 \leq \omega \leq \omega_{q}} M(\omega) \rightarrow 1\right)$.

Combining (18)-(20), we can conclude with the following closed-loop system stability condition for antiwindup design shown in Fig. 2:

$$
\left\|W_{1}(s) \tilde{\theta}_{1}(s)\right\|_{\infty}=\left\|\lambda(1+\varepsilon s) \tilde{\theta}_{1}(s)\right\|_{\infty}<1
$$

where $W_{1}(s)=\lambda(1+\varepsilon s), \lambda \geq 3 / 2$, and $\varepsilon \in\left(0,\left(3 \omega_{q}\right)^{-1}\right)$.

Remark 1: Due to the sufficiency of conditions (6) and (22), there are multiple choices of the antiwindup compensators $\theta_{1}(s)$ and $\theta_{2}(s)$. A straightforward solution is $\theta_{1}(s)=0$ and $\theta_{2}(s)=P(s)$, which corresponds to the classic internal model-based antiwindup. However, it is still an open problem to optimize the compensator design in the presence of system uncertainties for robustness considerations, which will be discussed in Section III-C.

\section{Antiwindup Robustness Condition}

We recall the fact that the scanning operations of nanostages are usually with various sample loads, which results in uncertainties of system dynamics. Therefore, it is crucial to consider the antiwindup robustness in the applications of nanoscanning.

We consider the plant model uncertainties in additive form described by $\Delta_{P}(s)$, which is bounded by the weighting function $W_{2}(s)$

$$
\left|\Delta_{P}(j \omega)\right|<\left|W_{2}(j \omega)\right| \quad \forall \omega \in \mathbb{R} .
$$

Accordingly, the actual plant model can be written as

$$
P_{\Delta}(s)=P(s)+\Delta_{P}(s) .
$$

By substituting the actual plant model into (6), we can derive

$$
\begin{aligned}
\theta_{2 \Delta}(s) & =P_{\Delta}(s)\left(1+\frac{\theta_{1}(s)}{1-Q(s) e^{-L s}}\right) \\
& =\left(P(s)+\Delta_{P}(s)\right)\left(1+\tilde{\theta}_{1}(s)\right) .
\end{aligned}
$$

Note that the antiwindup unit $\theta_{2}(s)$ designed for the nominal plant model $P(s)$ has an error of $\Delta_{\theta 2}(s)$

$$
\Delta_{\theta 2}(s)=\left|\theta_{2 \Delta}(s)-\theta_{2}(s)\right|=\left|\Delta_{P}(s)\left(1+\tilde{\theta}_{1}(s)\right)\right| .
$$

In order to eliminate the adverse effects caused by plant uncertainties on saturation compensation performance of the proposed control scheme shown in Fig. 2, the design of the antiwindup compensators should satisfy [29]

$$
\left\|W_{2}(s)\left(1+\tilde{\theta}_{1}(s)\right)\right\|_{\infty} \ll 1 .
$$

\section{D. $H_{\infty}$ Optimization of Antiwindup Compensators}

Combining the conditions (22) and (27) for the antiwindup compensators, we formulate the antiwindup design as the following $H_{\infty}$ optimization problem for the time-delay system:

$$
\gamma_{\mathrm{opt}}=\inf _{\theta_{1}(s) \in \mathcal{H}_{\infty}}\left\|\left[\begin{array}{c}
W_{1}(s) \tilde{\theta}_{1}(s) \\
W_{2}(s)\left(1+\tilde{\theta}_{1}(s)\right)
\end{array}\right]\right\|_{\infty}
$$

where $\gamma_{\text {opt }}$ is the optimal index.

We further define

$$
\begin{aligned}
& G(s)=1, \\
& C(s)=-\frac{\tilde{\theta}_{1}(s)}{1+\tilde{\theta}_{1}(s)} .
\end{aligned}
$$

The $H_{\infty}$ optimization problem (28) can be transformed into the following mixed sensitivity optimization problem:

$$
\gamma_{\mathrm{opt}}=\inf _{C(s) \in \mathcal{H}_{\infty}}\left\|\left[\begin{array}{c}
W_{s}(s)(1+G(s) C(s))^{-1} \\
W_{t}(s) G(s) C(s)(1+G(s) C(s))^{-1}
\end{array}\right]\right\|_{\infty}
$$

where

$$
\begin{aligned}
& W_{s}(s)=W_{2}(s) \\
& W_{t}(s)=-W_{1}(s) .
\end{aligned}
$$

The standard $H_{\infty}$ control theory, as readily implemented in the robust control toolbox of MATLAB, can now be applied to this particular problem. The optimal filter $C(s)$ can be achieved based on the optimal $H_{\infty}$ index $\gamma_{\text {opt }}$.

Correspondingly, the optimized antiwindup compensators can be further derived as

$$
\begin{aligned}
& \theta_{1}(s)=-\frac{C(s)\left(1-Q(s) e^{-L s}\right)}{1+C(s)} \\
& \theta_{2}(s)=P(s)\left(1-\frac{C(s)}{1+C(s)}\right) .
\end{aligned}
$$

Remark 2: Note that the theoretical analysis supporting the proposed antiwindup compensator design is based on the requirements on $B(s) P(s)$ and $Q(s)$, obeying (1)-(4). In real applications, appropriate nonideal low-pass filters can be implemented [as opposed to the ideal case of (1)-(4)] to derive the antiwindup compensation for repetitive control structures. In the application to nanostage (see Section IV), we will provide the design and implementation details, where the experimental results also demonstrate the stability and performance with the low-pass filter approximation. 

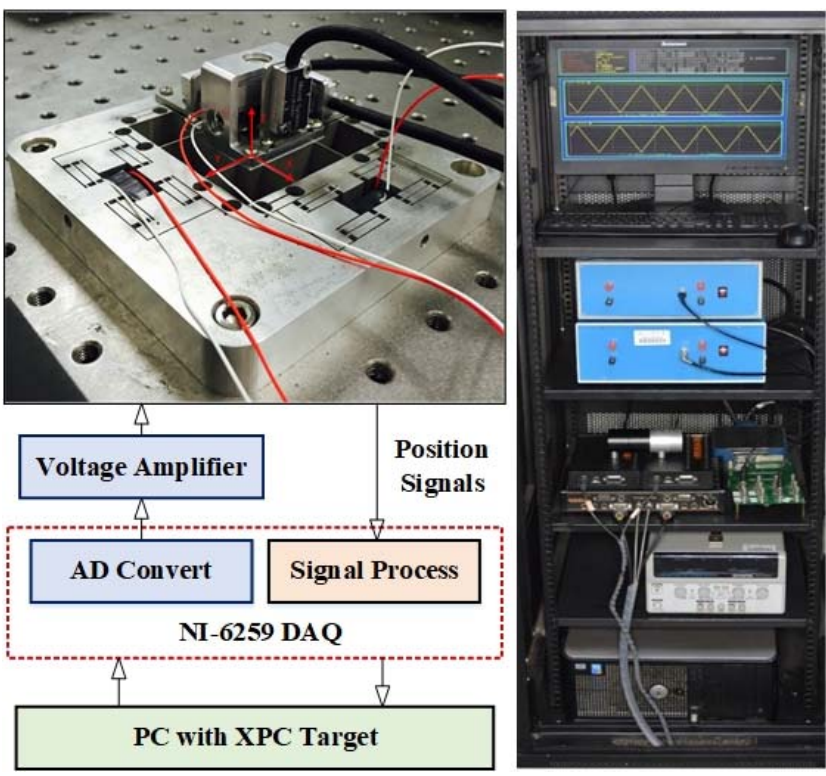

Fig. 6. Experimental setup of a piezoelectric-actuator-driven nanostage.

\section{Application to A Nanostage}

In this section, the proposed predictive antiwindup design for repetitive control structure is evaluated using real-time experiments on a piezoelectric actuator-driven nanostage.

\section{A. Experimental Setup and Model Identification}

We first briefly describe the experimental apparatus, as shown in Fig. 6. The proposed flexure-based XYZ piezoelectric nanostage consists of a parallel $\mathrm{XY}$ nanostage and a $\mathrm{Z}$ nanostage mounted onto the $\mathrm{XY}$ platform. For more details of the mechanical design, please refer to the previous result [33]. The design motivation of the XYZ nanostage is to support the ultramicroscopic imaging of surface plasmon confocal microscope [33], where the XYZ nanostage adjusts the sample along the $Z$-direction to the focal plane and then carries the sample to accomplish the $X Y$ planar scanning by following triangle-wave signals. Note that we will focus on the scanning motion of the $\mathrm{XY}$ stage, where the Z-stage is serving as a constant load for the parallel $\mathrm{XY}$ stage. Due to the symmetric design in the $X$-and $Y$-directions, we will limit the following experiments on the $X$-direction only, while the couplings of $\mathrm{X}-\mathrm{Y}$ motion are considered as disturbances to the control system of the $X$-direction.

The machined nanostage is mounted on an air-floatation platform to reduce the external effects, including vibrations and disturbances on the experimental results. Two piezoelectric actuators are used to drive the nanostage to achieve planar translations. Considering the high bandwidth and high precision requirements, high bandwidth voltage amplifiers are designed to drive the piezoelectric actuators. Accordingly, linear encoders (from MicroE Systems) with a resolution of $1.2 \mathrm{~nm}$ are instrumented as the displacement sensors to generate real-time position signals. Feedback control implementations are deployed using MATLAB/Simulink real-time
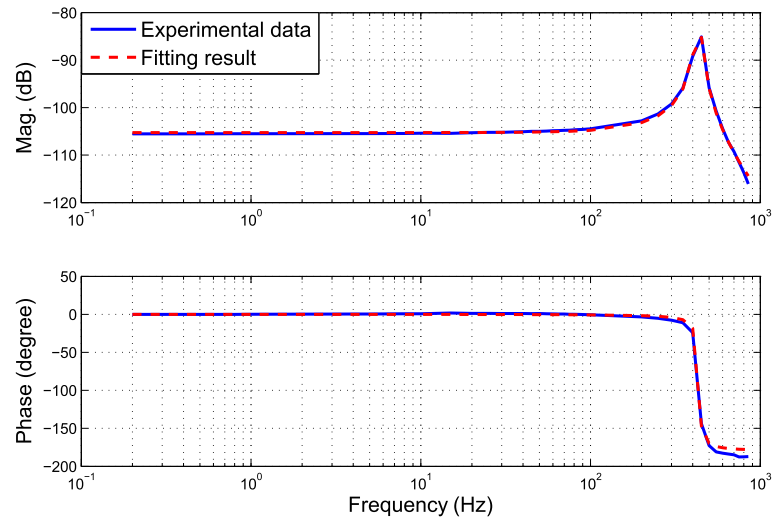

Fig. 7. Frequency responses of the plant.

control package xPCTarget with National Instruments (NI) PCI-6259 I/O hardware with a sampling frequency of $20 \mathrm{kHz}$.

By applying swept sine signals ranging from 0.2 to $850 \mathrm{~Hz}$, we can obtain the corresponding frequency response data (blue solid line in Fig. 7), with which the plant model can be identified by a second-order model (red dashed line in Fig. 7)

$$
P(s)=\frac{40.28}{s^{2}+141.21 s+7.38 \times 10^{6}} .
$$

\section{B. Controller Design}

We would like to sketch the design of the proposed control architecture by studying a $50-\mathrm{Hz}$ triangular wave tracking example of the nanostage, which is representative in highspeed-scanning applications. Therefore, the repetitive controller is designed with the parameter $L=0.02$. Referring to [10] and [11], we design $Q(s)$ and $B(s)$ in the following implementable forms as:

$$
\begin{aligned}
& B(s)=\frac{P^{-1}(s)}{(\tau s+1)^{n}}=\frac{s^{2}+141.21 s+7.38 \times 10^{6}}{40.28\left(\frac{1}{2000 \pi} s+1\right)^{2}} \\
& Q(s)=\frac{1}{\alpha \tau s+1}=\frac{1}{\frac{1}{400 \pi}+1} .
\end{aligned}
$$

Recall the $H_{\infty}$ optimization problem for the antiwindup design. We need to determine the weighting functions $W_{1}(s)$ and $W_{2}(s)$ to design the antiwindup compensators. Particularly, the weighting function $W_{2}(s)$ can be experimentally determined by observing the discrepancy between the actual dynamics (measured experimentally) and the nominal model. The weighting function $W_{1}(s)$ is determined by the parameters $\lambda$ and $\varepsilon$, where $\lambda \geq 3 / 2,0<\varepsilon<1 /\left(3 \omega_{q}\right)$, and $\omega_{q}=400 \pi$. In this design, we select $\lambda=2, \varepsilon=1 \times 10^{-4}$, and $W_{2}(s)$ to have high pass to accommodate system uncertainties at high frequencies. Accordingly, $W_{1}(s)$ and $W_{2}(s)$ are determined as

$$
\begin{aligned}
& W_{1}(s)=0.0002 s+2, \\
& W_{2}(s)=\frac{1.51 \times 10^{-2} s^{2}+18.85 s+2.37 \times 10^{4}}{s^{2}+304.62 s+9.10 \times 10^{6}} .
\end{aligned}
$$

We derive the optimal filter $C(s)$ with index $\gamma_{\text {opt }}=1.44 \times$ $10^{-4}$ by utilizing the MATLAB function "mixsyn"

$$
C(s)=\frac{2.25(s+100)\left(s^{2}+191.51 s+1.29 \times 10^{7}\right)}{\left(s+1.82 \times 10^{5}\right)\left(s^{2}+304.62 s+9.10 \times 10^{6}\right)} .
$$



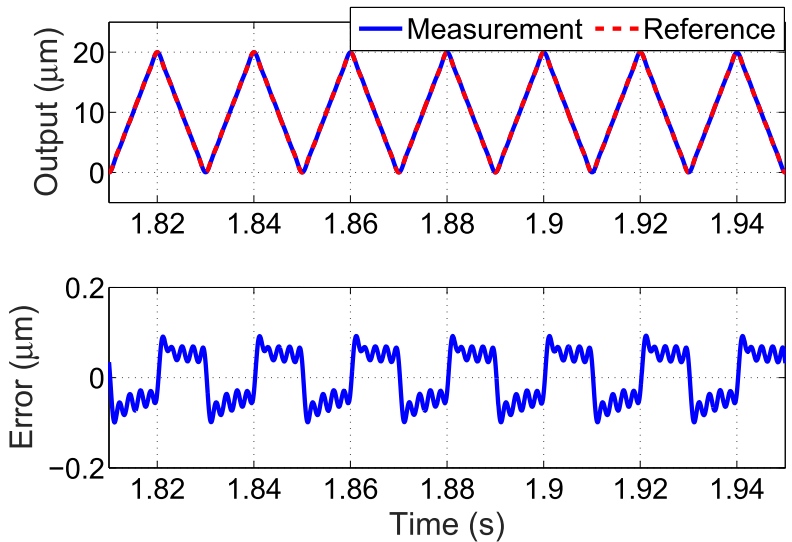

Fig. 8. Experimental results of tracking a $50-\mathrm{Hz}$ triangular wave without saturation.

Note that the optimal filter $C(s)$ exhibits the feature of a high-pass filter, whose gain trends to 0 at the low-frequency range and turns to be greater than 1 at the high-frequency range, due to the effect of plant uncertainties. The small value of $\gamma_{\text {opt }}$ is obtained due to the accuracy of the identified model. As a matter of fact, a larger system uncertainty will lead to a higher gain of $C(s)$. By substituting the optimal filter $C(s)$ into (33) and (34), we further derive the antiwindup compensators as (41) and (42), as shown at the bottom of the page.

\section{Experimental Results}

We start with the case of tracking a $50-\mathrm{Hz}$ triangular wave without saturation. As clearly shown in Fig. 8, the designed repetitive controller demonstrates excellent tracking performance, where the maximum error (ME) is $99.01 \mathrm{~nm}$ and the root-mean-square error (RMSE) is $58.11 \mathrm{~nm}$.

We further increase the tracking range of the piezoelectric-driven nanostage to $55 \mu \mathrm{m}$ in order to trigger the actuator saturation to verify the antiwindup performance of the proposed predictive antiwindup method. Note that due to the characteristics of piezo actuators, only the positive voltage can be applied. Accordingly, we set the saturation limits as $\sigma_{1}=0$ and $\sigma_{2}=5$ such that the driving voltage applied to the piezo-driven nanostage is limited in $0 \sim 100 \mathrm{~V}$. As shown in Fig. 9, the designed repetitive controller cannot guarantee the stability of the closed-loop system due to the large initial controller outputs, where huge oscillations of system outputs are observed. With the action of the antiwindup compensators, the commanded inputs converge and the system outputs achieve asymptotic tracking, as shown in Fig. 9.

It is also interesting to compare the proposed anticipatory antiwindup method with the traditional immediate antiwindup

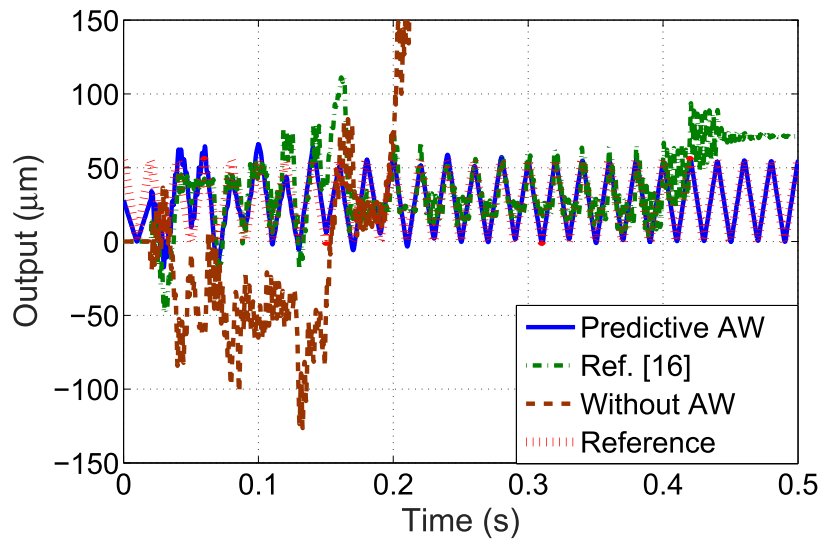

Fig. 9. Tracking of $50-\mathrm{Hz}$ triangular wave with an amplitude of $55 \mu \mathrm{m}$.
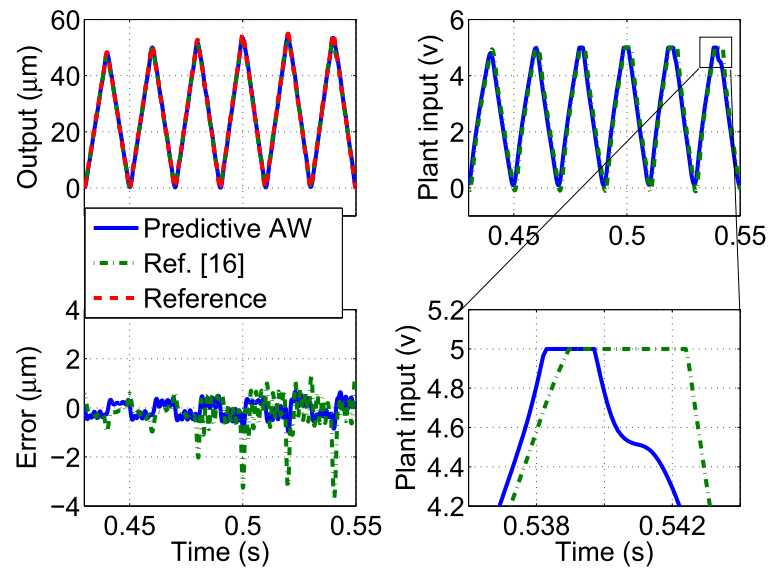

Fig. 10. Tracking of $50-\mathrm{Hz}$ triangular wave with gradually increasing amplitude.

strategy. Particularly, we designed the well-known immediate antiwindup compensator on top of the repetitive controller according to the results in [16], for the same tracking problem. As shown in Fig. 9, the designed immediate antiwindup compensator cannot handle the saturation well because the initial controller outputs are saturated severely. However, the repetitive controller with immediate antiwindup compensator can guarantee the stability of the closed-loop system if the amplitude of triangular signals gradually increases to $55 \mu \mathrm{m}$, as shown in Fig. 10. The comparison results demonstrate that the predictive antiwindup method significantly improves the transient performance (including convergence speed, saturation recovery time, and RMSE) than the immediate antiwindup scheme. As listed in Table I, the proposed predictive antiwindup compensation improves the tracking accuracy by $49.62 \%$ compared with the immediate antiwindup scheme.

$$
\begin{aligned}
& \theta_{1}(s)=-\frac{0.69(s+100)\left(s^{2}+191.51 s+1.29 \times 10^{7}\right)}{\left(s+5.58 \times 10^{4}\right)\left(s^{2}+351.52 s+9.12 \times 10^{6}\right)}\left(1-\frac{e^{-0.02 s}}{7.96 \times 10^{-4} s+1}\right) \\
& \theta_{2}(s)=\frac{68.17\left(s+3.29 \times 10^{4}\right)\left(s^{2}+398.03 s+9.16 \times 10^{6}\right)}{\left(s+5.58 \times 10^{4}\right)\left(s^{2}+141.21 s+7.38 \times 10^{6}\right)\left(s^{2}+351.52 s+9.12 \times 10^{6}\right)}
\end{aligned}
$$


TABLE I

TraCking ERror of Different Control StruCtures

\begin{tabular}{llllll}
\hline \hline Controller & $\begin{array}{l}\text { Predictive } \\
\text { anti-windup }\end{array}$ & $\begin{array}{l}\text { Conventional } \\
\text { anti-windup } \\
\text { in [16] }\end{array}$ & Ref. [15] & Ref. [22] & Ref. [23] \\
\hline $\begin{array}{l}\text { RMSE } \\
(\mu \mathrm{m})\end{array}$ & 0.66 & 1.31 & 2.39 & 3.42 & 3.54 \\
\hline \hline
\end{tabular}
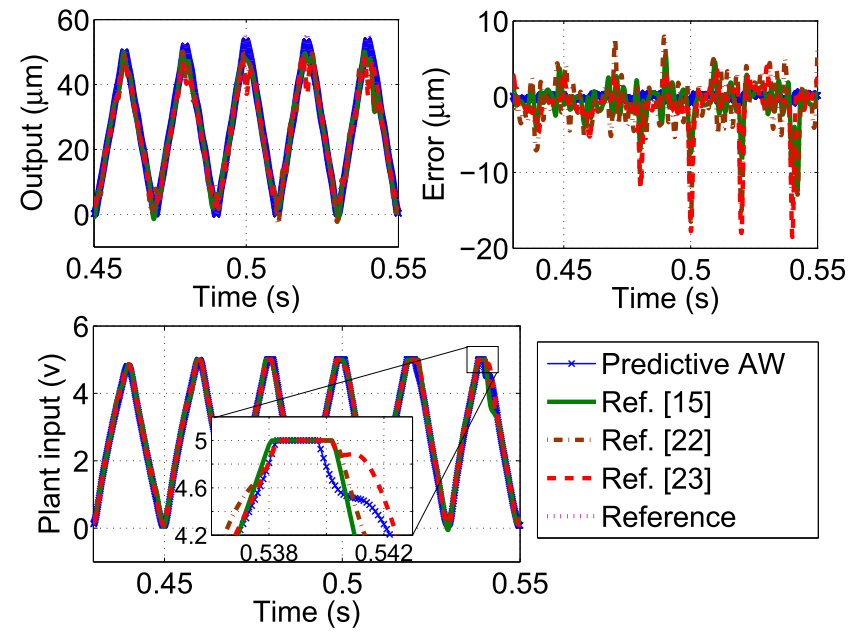

Fig. 11. Comparisons between the proposed control structure and the existing control methods.

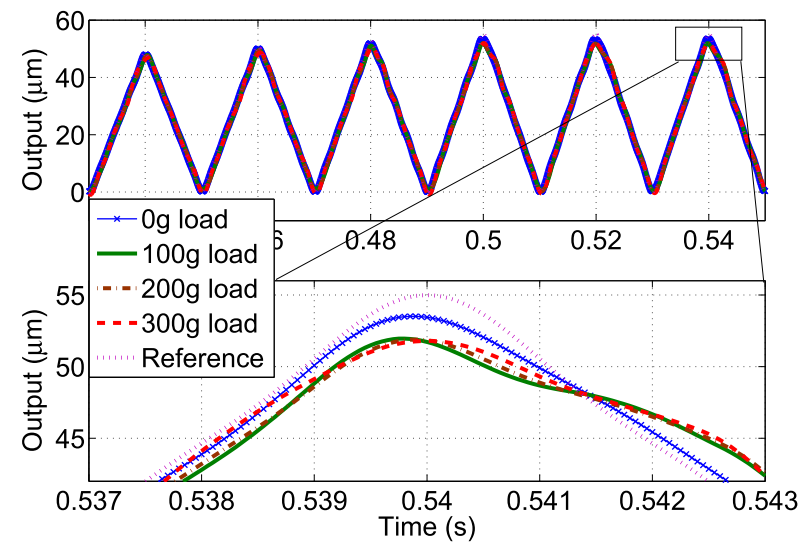

Fig. 12. Tracking of 50-Hz triangular wave in cases of different loads.

Moreover, the existing repetitive antiwindup control methods [15], [22], [23] are also deployed on the nanostage for comparisons. Although these control methods can guarantee the tracking performance in the presence of actuator saturation, the proposed repetitive control structure with predictive antiwindup compensation demonstrates the minimum tracking error, as well as the least saturation recovery time, as shown in Fig. 11. Compared with the control methods proposed in [15], [22], [23], the proposed control structure improves the RMSE by $72.38 \%, 80.70 \%$, and $81.36 \%$, respectively, as shown in Table I.

To further verify the robustness of the proposed predictive antiwindup architecture, different loads (ranging from 0 to $300 \mathrm{~g}$ ) are imposed on the nanostage to change the system dynamics. From Fig. 12, it is straightforward that the repetitive
TABLE II

Root-MEAN-SQUARE TRACKING ERRor With DifFERENT LoADS

\begin{tabular}{ccccc}
\hline \hline Load & $0 \mathrm{~g}$ & $100 \mathrm{~g}$ & $200 \mathrm{~g}$ & $300 \mathrm{~g}$ \\
\hline RMSE $(\mu \mathrm{m})$ & 0.66 & 0.74 & 0.84 & 0.97 \\
\hline \hline
\end{tabular}

TABLE III

EXECUTION TIME COMPARISON OF DiFFERENT CONTROL STRUCTURES

\begin{tabular}{llll}
\hline \hline Algorithm & MinTET (s) & MaxTET (s) & AvgTET (s) \\
\hline $\begin{array}{l}\text { Predictive } \\
\text { anti-windup }\end{array}$ & $3.76 \times 10^{-6}$ & $1.65 \times 10^{-5}$ & $5.93 \times 10^{-6}$ \\
$\begin{array}{l}\text { Conventional } \\
\text { anti-windup in }\end{array}$ & $2.86 \times 10^{-6}$ & $1.39 \times 10^{-5}$ & $4.99 \times 10^{-6}$ \\
Ref. [16] & & & \\
\hline \hline
\end{tabular}

control-based predictive antiwindup tracking architecture can handle both saturation well under the influence of system uncertainties such that good performance with an RMSE less than $0.97 \mu \mathrm{m}$ is achieved, as listed in Table II.

It is also interesting to check the implementation complexity of the proposed algorithm compared with other algorithms. Therefore, we collected the execution time of the controllers in the process of real-time control implementations. As depicted in Table III, the average execution time (AvgTET) of the proposed scheme is increased by around 19\% compared with the conventional antiwindup scheme in [16], which is quite acceptable for implementations.

Remark 3: Note that due to the hardware configurations, all the experiment results are based on one side saturation (positive voltages), which is the common practice in nanoscanning applications. However, the proposed predictive antiwindup method is applicable to two-side saturation (with positive and negative control inputs) shown in Fig. 5.

\section{CONClusion}

Large-range nanoscannings with repetitive controllers are prone to windup problems due to the existence of multiple integrators and the limited travel range of the microactuators, which may result in significant performance drop and stability issues. We proposed a predictive antiwindup design on top of the conventional repetitive control structure to deal with the saturation problem. Due to the time-delay block in the control loop (to reflect the periodic reference), the time-lead controller output was obtained such that an additional saturation function was introduced to activate the antiwindup compensators in anticipation of actuator saturation. According to the I/O-based equivalent representation and sector bound criterion, stability conditions for the saturated closed-loop system were provided, where the design of robust antiwindup compensators was formulated as an $H_{\infty}$ optimization problem that can be solved by mixed sensitivity optimization. Real-time experiments of the proposed method on a piezo-driven nanostage demonstrated significant improvement of the saturation compensation capability and tracking performance, outperforming representative existing results. 


\section{REFERENCES}

[1] C. Zhang, J. Shi, W. Wang, N. Xi, Y. Wang, and L. Liu, "Simultaneous measurement of multiple mechanical properties of single cells using AFM by indentation and vibration," IEEE Trans. Biomed. Eng., vol. 64, no. 12, pp. 2771-2780, Dec. 2017.

[2] F. Tajaddodianfar, S. O. R. Moheimani, and J. N. Randall, "Scanning tunneling microscope control: A self-tuning PI controller based on online local barrier height estimation," IEEE Trans. Control Syst. Technol., vol. 27, no. 5, pp. 2004-2015, Sep. 2019.

[3] B. Zhang, S. Pechprasarn, J. Zhang, and M. G. Somekh, "Confocal surface plasmon microscopy with pupil function engineering," Opt. Express, vol. 20, no. 7, pp. 7388-7397, Jun. 2012.

[4] S. Devasia, E. Eleftheriou, and S. O. R. Moheimani, "A survey of control issues in nanopositioning," IEEE Trans. Control Syst. Technol., vol. 15, no. 5, pp. 802-823, Sep. 2007.

[5] G.-Y. Gu, L.-M. Zhu, C.-Y. Su, H. Ding, and S. Fatikow, "Modeling and control of piezo-actuated nanopositioning stages: A survey," IEEE Trans. Autom. Sci. Eng., vol. 13, no. 1, pp. 313-332, Jan. 2016.

[6] X. Chen, C.-Y. Su, Z. Li, and F. Yang, "Design of implementable adaptive control for micro/nano positioning system driven by piezoelectric actuator," IEEE Trans. Ind. Electron., vol. 63, no. 10, pp. 6471-6481, Oct. 2016.

[7] J. Chen, B. Ren, and Q.-C. Zhong, "UDE-based trajectory tracking control of piezoelectric stages," IEEE Trans. Ind. Electron., vol. 63, no. 10, pp. 6450-6459, Oct. 2016.

[8] J. P. Mishra, Q. Xu, X. Yu, and M. Jalili, "Precision position tracking for piezoelectric-driven motion system using continuous third-order sliding mode control," IEEE/ASME Trans. Mechatronics, vol. 23, no. 4 , pp. 1521-1531, Aug. 2018.

[9] Y. Jian, D. Huang, J. Liu, and D. Min, "High-precision tracking of piezoelectric actuator using iterative learning control and direct inverse compensation of hysteresis," IEEE Trans. Ind. Electron., vol. 66, no. 1, pp. 368-377, Jan. 2019.

[10] S. Hara, Y. Yamamoto, T. Omata, and M. Nakano, "Repetitive control system: A new type servo system for periodic exogenous signals," IEEE Trans. Autom. Control, vol. AC-33, no. 7, pp. 659-668, Jul. 1988.

[11] T. E. Peery and H. Özbay, " $H_{\infty}$ optimal repetitive controller design for stable plants," ASME J. Dyn. Syst., Meas., Control, vol. 119, no. 3, pp. 541-547, Sep. 1997.

[12] R. Cao and K.-S. Low, "A repetitive model predictive control approach for precision tracking of a linear motion system," IEEE Trans. Ind. Electron., vol. 56, no. 6, pp. 1955-1962, Jun. 2009.

[13] Y. Li and Q. Xu, "Design and robust repetitive control of a new parallelkinematic XY piezostage for micro/nanomanipulation," IEEE/ASME Trans. Mechatronics, vol. 17, no. 6, pp. 1120-1132, Dec. 2012

[14] C.-X. Li, G.-Y. Gu, M.-J. Yang, and L.-M. Zhu, "High-speed tracking of a nanopositioning stage using modified repetitive control," IEEE Trans. Autom. Sci. Eng., vol. 14, no. 3, pp. 1467-1477, Jul. 2017.

[15] D. Sbarbaro, M. Tomizuka, and B. L. de la Barra, "Repetitive control system under actuator saturation and windup prevention," J. Dyn. Syst., Meas., Control, vol. 131, no. 4, Jul. 2009, Art. no. 044505.

[16] P. F. Weston and I. Postlethwaite, "Linear conditioning for systems containing saturating actuators," Automatica, vol. 36, no. 9, pp. 1347-1354, Sep. 2000
[17] B. Zhou, G.-R. Duan, and Z. Lin, "A parametric periodic Lyapunov equation with application in semi-global stabilization of discrete-time periodic systems subject to actuator saturation," Automatica, vol. 47, no. 2, pp. 316-325, Feb. 2011.

[18] J. Zheng and M. Fu, "Saturation control of a piezoelectric actuator for fast settling-time performance," IEEE Trans. Control Syst. Technol., vol. 21, no. 1, pp. 220-228, Jan. 2013.

[19] X. Wu and Z. Lin, "Dynamic anti-windup design in anticipation of actuator saturation," Int. J. Robust Nonlinear Control, vol. 24, no. 2, pp. 295-312, Jan. 2014.

[20] N. A. Ofodile and M. C. Turner, "Decentralized approaches to antiwindup design with application to quadrotor unmanned aerial vehicles," IEEE Trans. Control Syst. Technol., vol. 24, no. 6, pp. 1980-1992, Nov. 2016.

[21] S. Gayadeen and S. R. Duncan, "Discrete-time anti-windup compensation for synchrotron electron beam controllers with rate constrained actuators," Automatica, vol. 67, pp. 224-232, May 2016.

[22] J. V. Flores, J. M. G. da Silva, L. F. A. Pereira, and D. G. Sbarbaro, "Repetitive control design for MIMO systems with saturating actuators," IEEE Trans. Autom. Control, vol. 57, no. 1, pp. 192-198, Jan. 2012.

[23] J. V. Flores, J. M. G. da Silva, D. Sbarbaro, M. C. Turner, and A. T. Salton, "Antiwindup design for zero-phase repetitive controllers," J. Dyn. Syst., Meas., Control, vol. 137, no. 9, Sep. 2015 Art. no. 094503.

[24] S. Sajjadi-Kia and F. Jabbari, "Modified anti-windup compensators for stable plants," IEEE Trans. Autom. Control, vol. 54, no. 8, pp. 1934-1939, Aug. 2009.

[25] X. Wu and Z. Lin, "On immediate, delayed and anticipatory activation of anti-windup mechanism: Static anti-windup case," IEEE Trans. Autom. Control, vol. 57, no. 3, pp. 771-777, Mar. 2012.

[26] M. C. Turner, J. Sofrony, and G. Herrmann, "An alternative approach to anti-windup in anticipation of actuator saturation," Int. J. Robust Nonlinear Control, vol. 27, no. 6, pp. 963-980, Apr. 2017.

[27] A. Bazaei, Y. K. Yong, S. O. R. Moheimani, and A. Sebastian, "Tracking of triangular references using signal transformation for control of a novel AFM scanner stage," IEEE Trans. Control Syst. Technol., vol. 20, no. 2, pp. 453-464, Mar. 2012.

[28] C.-X. Li, G.-Y. Gu, L.-M. Zhu, and C.-Y. Su, "Odd-harmonic repetitive control for high-speed raster scanning of piezo-actuated nanopositioning stages with hysteresis nonlinearity," Sens. Actuators A, Phys., vol. 244 pp. 95-105, Jun. 2016

[29] P. Liu, P. Yan, Z. Zhang, and H. Ozbay, "Robust antiwindup compensation for high-precision tracking of a piezoelectric nanostage," IEEE Trans. Ind. Electron., vol. 63, no. 10, pp. 6460-6470, Oct. 2016.

[30] T. Hu, A. R. Teel, and L. Zaccarian, "Anti-windup synthesis for linear control systems with input saturation: Achieving regional, nonlinear performance," Automatica, vol. 44, no. 2, pp. 512-519, Feb. 2008.

[31] X. J. Li, "On the absolute stability of systems with time lags," Chin. Math., vol. 4, no. 4, pp. 609-626, Nov. 1963.

[32] P.-A. Bliman, "Lyapunov-Krasovskii functionals and frequency domain: Delay-independent absolute stability criteria for delay systems," Int. J. Robust Nonlinear Control, vol. 11, no. 8, pp. 771-788, Jul. 2001.

[33] P. Liu, P. Yan, and H. Özbay, "Design and trajectory tracking control of a piezoelectric nano-manipulator with actuator saturations," Mech. Syst. Signal Process., vol. 111, pp. 529-544, Oct. 2018. 